\title{
Physical therapy for old-timer athletes with chronic back pain
}

\author{
DOI: https://doi.org/10.5114/pq.2020.92475
}

\author{
Alexey Honcharov', Larisa Ruban', Andrey Litovchenko', Daria Okun', Artem Turchinov \\ ${ }^{1}$ Kharkiv State Academy of Physical Culture, Kharkiv, Ukraine \\ ${ }^{2}$ National Academy of National Guard of Ukraine, Kharkiv, Ukraine
}

\section{Abstract}

Introduction. The purpose of the study was to assess the effectiveness of a physical therapy program for old-timer athletes with chronic back pain, which is a common complaint in this group, especially among wrestlers. It results from the fact that in wrestlers, the load is usually borne by a specific muscle group. Recent data on the effectiveness of the treatment and prevention of chronic back pain among old-timer athletes have shown a significant role of physical therapy. Increasingly used in dystrophic diseases of the spine, it is economically justified, does not require special conditions, and is not accompanied by complications. The only question that remains is what actions are rationally applied, depending on clinical manifestations.

Methods. The study involved analysis of current literature on the visual analogue scale for Oswestry questionnaire, research methods for spinal motor properties described by Weiss and Zembaty, Schober test, Thomayer test, methods of mathematical statistics.

Results. A physical therapy program was developed which was prescribed for 3 periods: muscle relaxation, myocorrection, and myotonization; they differed in motor activity, direction, and means of physical therapy. The physical therapy means included morning hygienic gymnastics, post-isometric relaxation, hydrokinesiotherapy with traction elements, kinesiotherapy with Pilates elements, phonophoresis with hydrocortisone, massage. The program improved spinal mobility and life quality in old-timer athletes with chronic back pain.

Conclusions. The physical therapy program for old-timer wrestlers statistically significantly improved the empirical parameters and quality of life, reduced as a result of chronic back pain.

Key words: chronic pain, old-timer sports, wrestlers, kinesiotherapy, traction influence

\section{Introduction}

Chronic back pain, especially in the lumbosacral spine, in old-timer athletes, particularly wrestlers, appears as a consequence of the fact that the load is usually borne by a certain muscle group. The development of myofascial pain syndromes and the changes identified in the affected areas trigger the dystrophic and degenerative processes in the spine. Oldtimer athletes usually present no pain in areas with dystrophic changes in the vertebrae and discs, and, on the contrary, there are pains in areas with no dystrophic changes but affected by spastic muscle conditions [1, 2].

Physical therapy holds one of the leading places in the rehabilitation system of patients with chronic back pain, with the following tasks: normalizing the central nervous system tone; increasing blood and lymph flow in the lumbosacral spine; promoting muscle relaxation; improving the function of all body systems and preventing relapse; forming the muscle corset of the spine. Old-timer athletes, at the end of the course of physical therapy, have an opportunity to be fully adapted to everyday and uncomplicated professional activities [3-5].

According to systematic reviews from sources such as MEDLINE-NLM, MEDLINE-EBSCO, Scopus Elsevier, Cochrane, DOAJ, SciELO, and PLOS ONE, the advance of studies in the treatment of chronic back pain indicates exercise application. The literature widely covers the use of aerobic exercises, such as walking, hydrokinesiotherapy, exercise on a bicycle ergometer. To develop flexibility and balance, aerobic and strength exercises are recommended, in particular, breathing exercises, stabilization stretching, aerobic fitness, and physiotherapy [6, 7-11].
However, the works devoted to the integrated approach to physical therapy of old-timer wrestlers with chronic back pain are not enough for use in the training motor mode, they are fragmented and still leave unresolved problems in many aspects.

The purpose of the study was to assess the effectiveness of a physical therapy program for old-timer athletes with chronic back pain on the basis of an investigation of empirical data dynamics and the parameters of the spine functional condition.

\section{Subjects and methods}

The study was conducted in cooperation with the City Specialized Children and Youth Sports School of Olympic Reserve Aquatics named after Yana Klochkova and the Pioneer sports school in Kharkiv. It involved 34 athletes - oldtimers aged $36-45$ years, involved in the sport of wrestling. The study population was divided into 2 groups: the research group (Gr1; $n=19)$ and the comparison group ( $\mathrm{Gr} 2 ; n=15)$. As for the severity of vertebrogenic pain syndrome (according to Antonov), I-II degree of pain syndrome was diagnosed in all old-timer wrestlers. Degree 2 of muscle tension was established in $100 \%$ tested in kinaesthetic studies, which indicated the presence of the trigger stage of the neurodystrophic process.

When constructing the physical therapy program, we adhered to the principles of the formation of a kinesiotherapy program for degenerative dystrophic processes in the joints [12]. Physical therapy for Gr1 old-timers was prescribed in three periods [13], which differed in physical activity, direc-

Correspondence address: Larisa Ruban, Kharkiv State Academy of Physical Culture, Klochkovskaya 99, Kharkiv, 61058, Ukraine, e-mail: dariaokun@gmail.com 
tion, and means of physical therapy. During the morning relaxation period, $\mathrm{Gr} 1$ old-timer wrestlers performed a hygienic gymnastics complex (20 minutes), which ended with exercises in post-isometric relaxation (10 minutes). In the afternoon, every other day, hydrokinesiotherapy with traction elements (45 minutes) or kinesiotherapy with Pilates elements was performed, as well as exercises that increased the stability of the vestibular apparatus and coordination (40 minutes), phonophoresis with hydrocortisone (10 minutes), and then massage with the technique of Efimenko (30 minutes). A feature of the technique is that in a special part of the procedure, the main attention is paid to the condition of the massaged soft tissues in the zone of the lumbar spine. To eliminate hypertonicity of the lower back muscles, the muscle tissue is stretched along the edges with the removal technique. To do this, the fingertips penetrate under their outer edge, and these parts of the spine muscles are brought in with a smooth movement. After a pause of 2-3 seconds, the back muscles are released. After repeated stroking, the fingertips, with spiral or circular movements, rub the tendons of the back muscles and the sites of their attachment in the sacrum and crests of the ilium. During myocorrection in the training regimen in the morning, hygienic gymnastics was performed, with postisometric relaxation and psychocorrection at the end (40 minutes); in the afternoon, every other day, hydrokinesiotherapy with traction elements (45 minutes) or kinesiotherapy with $\mathrm{Pi}$ lates elements was performed, as well as correcting exercises. During the period of myotonization, Pilates gymnastics was used (30 minutes) instead of morning hygienic gymnastics; hydrokinesiotherapy with traction elements (35 minutes) or classes in the gym (60 minutes) in the evening were implemented every other day.

Gr2 individuals performed the morning hygienic gymnastics complex, which culminated in exercises in post-isometric relaxation. In the afternoon, the old-timer wrestlers of Gr2 participated in a kinesiotherapy complex in accordance with the generally accepted recommendations of the Ministry of Health of Ukraine, as well as in a session of therapeutic massage and phonophoresis with hydrocortisone.

To evaluate the effectiveness of the developed physical therapy program, the following research methods were used: analysis of modern scientific literature; questionnaires with the visual analogue scale, i.e. a segment of a straight $100-\mathrm{mm}$ line, whose starting point corresponds to the absence of pain and the end to unbearable pain; Oswestry questionnaire, which includes 10 sections describing various areas of life. The mobility of the lumbosacral spine was studied with the method of Weiss and Zembaty $[14,15]$. The movements of flexion and extension in the sagittal plane and with lateral inclinations in the frontal plane were determined. The Schober test and the Thomayer test served to study the mobility of the lumbar spine [14, 15]. Statistical processing was performed with the Statistica for Windows version 6.0 general-purpose data processing software package. The data are presented as arithmetic mean $(\bar{x})$, its error $(m)$, Student's confidence criterion $(t)$, degree of reliability $(p)$. For all analyses performed, the differences were considered significant at the level of $p<0.05$, where the minimum significance of differences was $95 \%$.

\section{Ethical approval}

The research related to human use has complied with all the relevant national regulations and institutional policies, has followed the tenets of the Declaration of Helsinki and the Council of Europe Convention on Human Rights and Biomedicine, and has been approved by the authors' institutional review board or an equivalent committee.

\section{Informed consent}

Informed consent has been obtained from all individuals included in this study.

\section{Results}

When comparing the dynamics of pain magnitudes as presented on the visual analogue scale, a statistically significant decrease in pain complaints was observed in Gr1 compared with $\mathrm{Gr} 2(t=-3.26 ; p<0.05)$ (Figure 1).

The Oswestry survey results showed a statistically significant decrease in pain complaints in various spheres of life among $\mathrm{Gr} 1$ old-timer wrestlers: the positive responses improved by 9.26 percentage points, as compared with 2.66 percentage points in Gr2. When comparing the indicator between groups, there was a statistically significant dynamics $(t=-7.78 ; p<0.05)$ (Figure 2).

The dynamics of the spine functional investigation revealed that the amplitude of the forward spine movement in

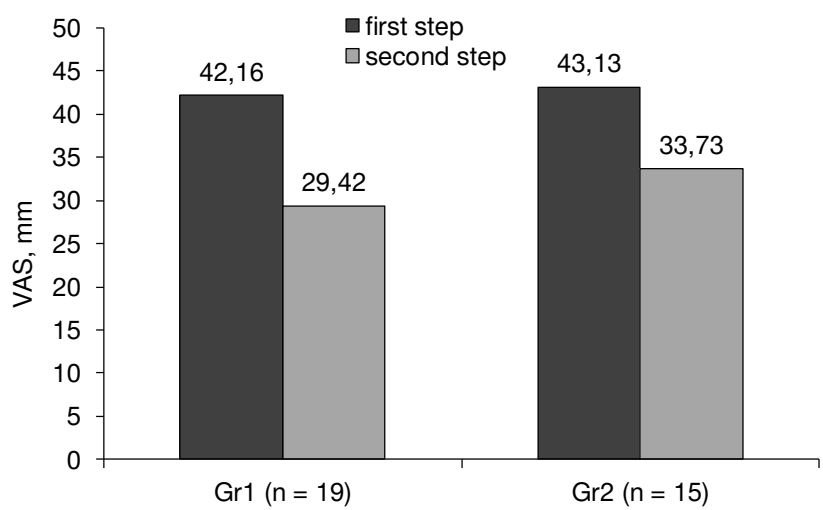

Figure 1. Dynamics of results for pain identified in the visual analogue scale (VAS) among old-timer wrestlers in the Gr1 and Gr2 study groups

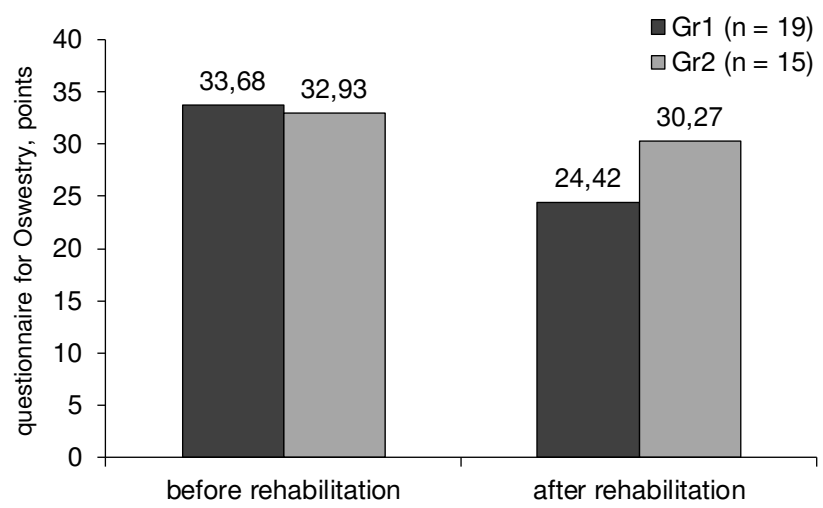

Figure 2. Dynamics of Oswestry questionnaire results among old-timer wrestlers in the Gr1 and Gr2 study groups

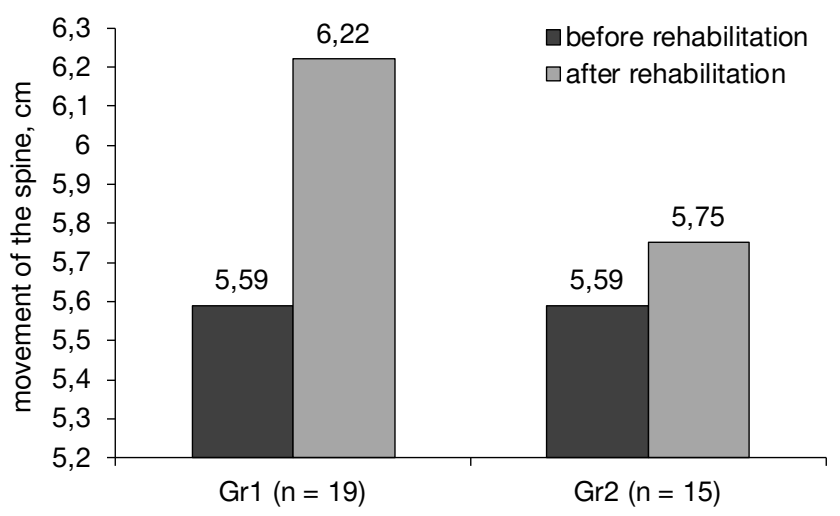

Figure 3. Dynamics of the amplitude of the forward movement of the spine in the sagittal plane among old-timer wrestlers in the Gr1 and Gr2 study groups 
the sagittal plane statistically significantly improved in Gr1 compared with Gr2 old-timer wrestlers $(t=2.07 ; p<0.05)$ (Figure 3).

The dynamics of the functional study of the spine implies that the amplitude of the backward movement of the spine in the sagittal plane statistically significantly improved in Gr1 compared with the same parameters in Gr2 old-timer wrestlers $(t=4.91 ; p<0.05)$ (Figure 4$)$.

The dynamics of the amplitude of the spine movement to the right in the frontal plane statistically significantly improved in $\mathrm{Gr} 1$ compared with the same parameters in $\mathrm{Gr} 2$ old-timer wrestlers $(t=4.70 ; p<0.05)$ (Figure 5).

The comparison of the amplitude of the spine movement to the left in the frontal plane showed a statistically significant improvement in Gr1 compared with Gr2 old-timer wrestlers $(t=2.74 ; p<0.05)$ (Figure 6).

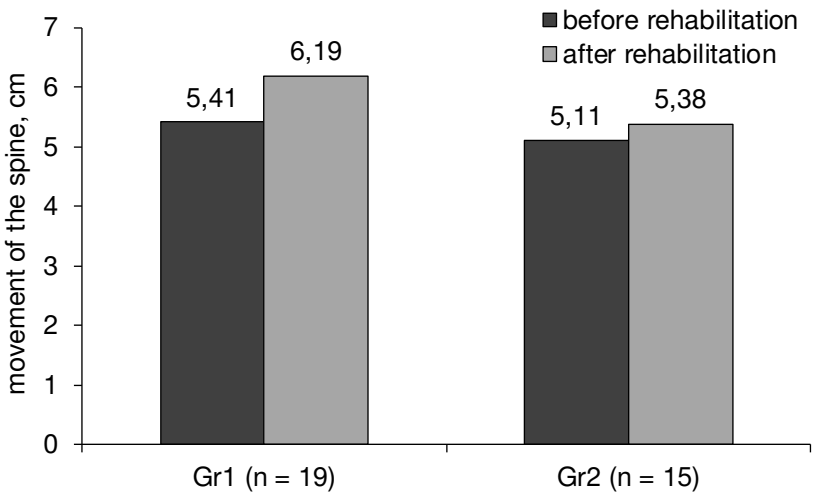

Figure 4. Dynamics of the amplitude of the back movement of the spine in the sagittal plane among old-timer wrestlers in the $\mathrm{Gr} 1$ and $\mathrm{Gr} 2$ study groups

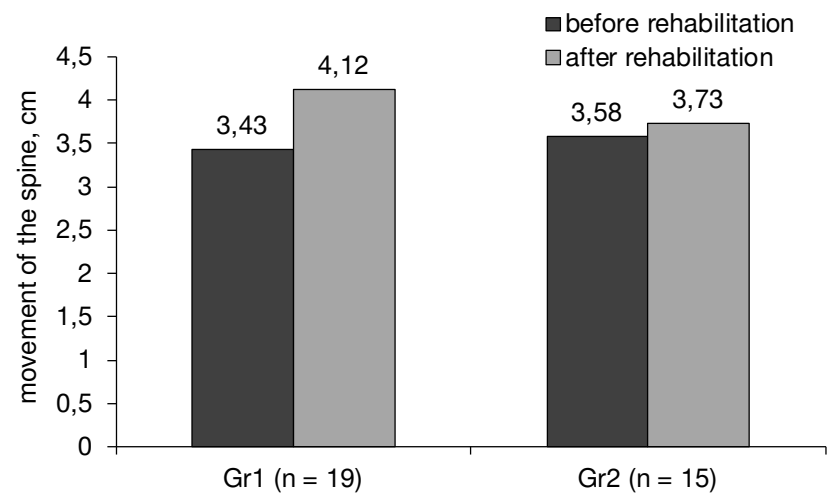

Figure 5. Dynamics of the amplitude of the spine movement to the right in the frontal plane among old-timer wrestlers in the $\mathrm{Gr} 1$ and $\mathrm{Gr} 2$ study groups

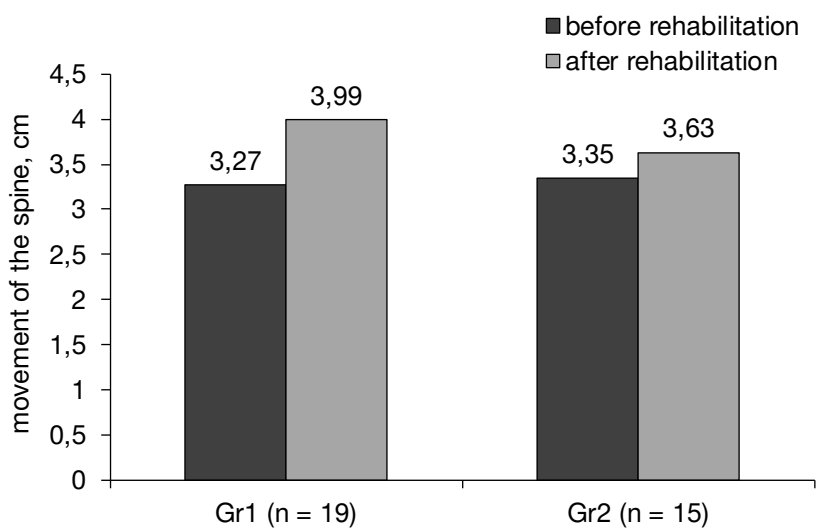

Figure. 6. Dynamics of the amplitude of the spine movement to

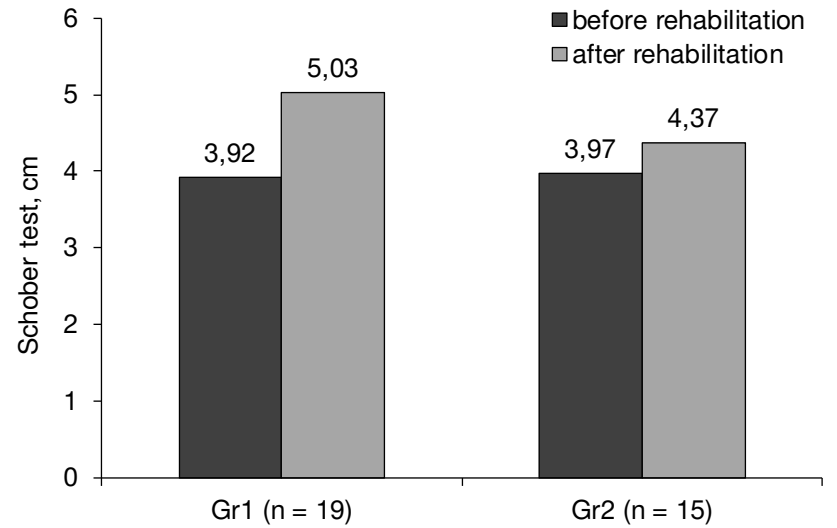

Figure 7. Dynamics of the Schober test among old-timer wrestlers in the $\mathrm{Gr} 1$ and $\mathrm{Gr} 2$ study groups

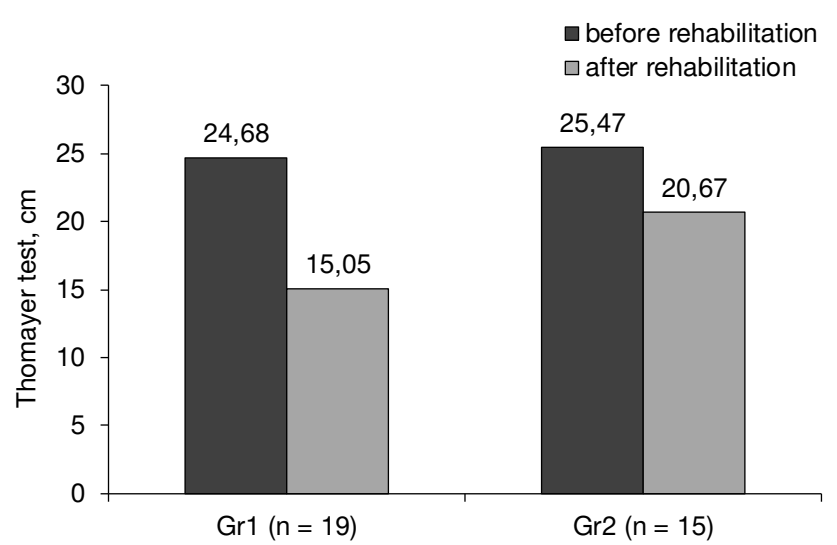

Figure 8. Dynamics of the Thomayer test among old-timer wrestlers in the Gr1 and Gr2 study groups

When comparing the indicator of the Schober test between the groups ( $\mathrm{Gr} 1$ and $\mathrm{Gr} 2$ ), a more pronounced dynamics was observed in $\mathrm{Gr} 1(t=2.96 ; p<0.05)$ (Figure 7).

Even more pronounced changes were noticed when analysing the parameters of the Thomayer test. The old-timer wrestlers in $\mathrm{Gr} 1$ presented a statistically significant decrease in the Thomayer test results in comparison with Gr2 $(t=7.65$; $p<0.05$ ) (Figure 8).

Under the influence of the complex rehabilitation measures, positive dynamics of diagnostic indicators of the functional state of the spine was observed in the tested persons of both groups. The comparison between groups revealed statistically significant changes in the old-timer wrestlers of Gr1. The results of the study confirmed the effectiveness of the physical therapy program that we developed: exercises in post-isometric relaxation and traction in water positively affected the increase in the amplitude of spine movement both in the sagittal and in the frontal plane.

\section{Discussion}

It can be noted that old-timer wrestlers present numerous neurological manifestations, especially in the lumbosacral spine, which is most often caused by damage to the intervertebral discs and, to a much lesser extent, by bone changes. The most common vertebrogenic cause of back pain in athletes is a sharp switch to a lower level of physical activity, for example, when they stop actively playing sports or discontinue professional physical training. According to the generally accepted views of the European countries on the directions in the treatment of chronic back pain, physical therapy constitutes its integral part $[16,17]$. 
A special task of kinesiotherapy in training motor mode is to promote the functional recovery of patients with chronic back pain. The main means of kinesiotherapy is exercise. When choosing physical exercises, one should bear in mind that they should not only improve blood and lymph flow in the spinal motion segment, but also reduce the emotional stress of sick athletes $[4,11,18]$. The use of post-isometric relaxation is the main indication for the widespread tension of the paravertebral muscles in vertebrogenic reflex syndromes [1, 7, 9, 16, 19].

Under the influence of a set of measures of physical therapy, positive dynamics of diagnostic indicators of the functional state of the spine was observed in the tested persons of both groups. The comparison between groups revealed statistically significant changes in the old-timer wrestlers of Gr1. The amplitudes of the forward $(t=2.07)$ and backward $(t=4.91)$ movement of the spine in the sagittal plane, as well as of the movement to the right $(t=4.70)$ and to the left $(t=2.74)$ in the frontal plane were statistically significantly improved among Gr1 individuals in comparison with the same parameters in old-timer wrestlers of Gr2 $(p<0.05$ for all indicators).

The obtained results confirm the findings of other authors $[2,6,8,10]$ on the use of exercises in water, which can be applied as an independent treatment for vertebral pain. Traction treatment in water helps to reduce pain, increase the height of the intervertebral foramen, intervertebral disc with horizontal traction. A special task of kinesiotherapy in training motor mode is to promote the functional recovery of patients $[3,6,11,17]$.

\section{Limitations}

The presented study has some limitations. Firstly, the small number of patients who were involved in the study and the inclusion of old-timer athletes only limit the ability to generalize the results to other populations. Secondly, the study was conducted within the framework of one sports specialization; this can also limit result generalization.

\section{Conclusions}

The findings of the study showed that the proposed physical therapy program for old-timer wrestlers contributed to the improvement of empirical parameters and quality of life reduced as a result of chronic back pain. Under the influence of the complex rehabilitation measures, positive dynamics of diagnostic indicators of the functional state of the spine was observed in the tested persons of both groups but the parameter changes in the group of old-timer athletes engaged in the authors' program reached statistical significance.

\section{Disclosure statement}

No author has any financial interest or received any financial benefit from this research.

\section{Conflict of interest}

The authors state no conflict of interest.

\section{References}

1. Fett $D$, Trompeter K, Platen P. Back pain in elite sports: a cross-sectional study on 1114 athletes. PLoS One. 2017;12(6):e0180130; doi: 10.1371/journal.pone.0180130.

2. Farahbakhsh F, Akbari-Fakhrabadi M, Shariat A, Cleland JA, Farahbakhsh F, Seif-Barghi T, et al. Neck pain and low back pain in relation to functional disability in different sport activities. J Exerc Rehabil. 2018;14(3): 509-515; doi: 10.12965/jer.1836220.110.

3. Coulter ID, Crawford C, Hurwitz EL, Vernon H, Khorsan R, Suttorp Booth $\mathrm{M}$, et al. Manipulation and mobilization for treating chronic low back pain: a systematic review and meta-analysis. Spine J. 2018;18(5):866-879; doi: 10.1016/j.spinee.2018.01.013.

4. Cuenca-Martínez F, Cortés-Amador S, Espí-López GV. Effectiveness of classic physical therapy proposals for chronic non-specific low back pain: a literature review. Phys Ther Res. 2018;21(1):16-22; doi: 10.1298/ptr.E9937.

5. Honcharov AG. Physical rehabilitation for lumbosacral spinal osteochondrosis in young men [in Ukrainian]. Moloda sportyvna nauka Ukrayiny: zb. nauk. pr. z haluzi fizychnoho vykhovannya i zdorovya lyudyny. 2014;18(3): 62-69.

6. Chenot JF, Greitemann B, Kladny B, Petzke F, Pfingsten M, Schorr SG. Non-specific low back pain. Dtsch Arztebl Int. 2017;114(51-52):883-890; doi: 10.3238/ arztebl.2017.0883.

7. Anand UA, Caroline PM, Arun B, Gomathi GL. A study to analyse the efficacy of modified Pilates based exercises and therapeutic exercises in individuals with chronic non specific low back pain: a randomized controlled trial. Int J Physiother Res. 2014;2(3):525-529.

8. Paolucci T, Attanasi C, Cecchini W, Marazzi A, Capobianco SV, Santilli V. Chronic low back pain and postural rehabilitation exercise: a literature review. J Pain Res. 2018;12:95-107; doi: 10.2147/JPR.S171729.

9. You JH, Kim SY, Oh DW, Chon SC. The effect of a novel core stabilization technique on managing patients with chronic low back pain: a randomized, controlled, experimenter-blinded study. Clin Rehabil. 2014;28(5):460-469; doi: 10.1177/0269215513506231.

10. Da Cruz FIM, Pinto RZ, Ferreira P, Lira FS. Low back pain, obesity, and inflammatory markers: exercise as potential treatment. J Exerc Rehabil. 2018;14(2):168-174; doi: 10.12965/jer.1836070.035.

11. Lin HT, Hung WC, Hung JL, Wu PS, Liaw LJ, Chang JH. Effects of Pilates on patients with chronic non-specific low back pain: a systematic review. J Phys Ther Sci. 2016;28(10):2961-2969; doi: 10.1589/jpts.28.2961.

12. Andreichuk AY. Methodological principles of physical therapy of patients with degenerative-dystrophic diseases of the musculoskeletal system [in Ukrainian]. Art of Medicine. 2018;3(7):174-177.

13. Mahdavi NR. Physical rehabilitation of patients with fibromyalgia of the lumbosacral spine [in Russian] (doctoral thesis). Moscow: Russian State University of Physical Education, Sport, Youth and Tourism; 2006.

14. Evseev VI. Biomechanics of degenerative-dystrophic diseases of the spine (osteochondrosis, spondylosis, spondylarthrosis): monograph [in Russian]. Moscow: Ruscience; 2017.

15. Kashuba VA. Biomechanics of posture: monograph [in Russian]. Moscow: Olympus; 2003.

16. Cho HY, Kim EH, Kim J. Effects of the CORE exercise program on pain and active range of motion in patients with chronic low back pain. J Phys Ther Sci. 2014;26(8): 1237-1240; doi: 10.1589/jpts.26.1237.

17. Iwamoto J, Sato $\mathrm{Y}$, Takeda T, Matsumoto H. Return to play after conservative treatment in athletes with symptomatic lumbar disc herniation: a practice-based observational study. Open Access J Sports Med. 2011;2:25-31; doi: 10.2147/OAJSM.S17523. 
18. Alrwaily M, Almutiri M, Schneider M. Assessment of variability in traction interventions for patients with low back pain: a systematic review. Chiropr Man Therap. 2018; 26:35; doi: 10.1186/s12998-018-0205-z.

19. Avramenko OM. Mechanotherapy in the rehabilitation treatment of patients with osteochondrosis of the lumbosacral spine [in Ukrainian]. Slobozans'kij naukovosportivnij visnik. 2013;5:16-18. 